\title{
Differential Expression of Pseudoconditioning and Sensitization by Siphon Responses in Aplysia: Novel Response Selection After Training
}

\author{
Mark T. Erickson and Edgar T. Walters \\ Department of Physiology and Cell Biology, University of Texas Medical School at Houston, Houston, Texas 77225
}

Nonassociative training with a noxious unconditioned stimulus (US) applied to the head or tail of freely moving Aplysia caused a qualitative change in siphon responses to midbody test stimulation, so that the midbody test responses came to resemble the unconditioned siphon response (UR) to the US when tested $1 \mathrm{~d}$ after exposure to the US. Such a nonassociative, US-induced transformation of test responses into responses resembling the UR has traditionally been termed "pseudoconditioning." Short-term pseudoconditioning was compared to sensitization and to habituation in a reduced preparation that used a photocell to distinguish "head-type" siphon responses from qualitatively different "tail-type" responses. Transformation of test responses (pseudoconditioning) was observed only when the type of preexisting alpha response to the midbody test stimulus was different from the UR. Sensitization, defined as a US-induced enhancement of the alpha response to the test stimulus, was observed when the initial alpha response and the UR were of the same type. General sensory facilitation was excluded as a critical mechanism for pseudoconditioning by the observation that the same midbody test response could be transformed to either a head-type or tail-type response, depending on the site of the US, and by the observation that simply increasing the intensity of the midbody test stimulus in the absence of a head or tail US did not produce similar response transformations. These studies demonstrate pseudoconditioning in a preparation amenable to analysis at the level of identified neurons, and draw attention to a distinctive and widespread form of behavioral modifiability that has been neglected by investigators of learning.

The neural mechanisms of 2 simple forms of learning, sensitization and habituation, have received considerable experimental attention (e.g., Groves and Thompson, 1970; Kandel, 1976). This interest reflects the relative tractability of the simplest forms of learning for cellular and molecular analysis (e.g., Kandel and Schwartz, 1982) and the likelihood that simple forms of learning may be closely related to aspects of more complex learning (Grant, 1943b; Razran, 1971; Hawkins and Kandel, 1984). A third form of simple learning, pseudoconditioning, has not yet

\footnotetext{
Received Sept. 4, 1987; accepted Dec. 21, 1987.

This work was supported by National Institutes of Mental Health Grant MH38726, National Institutes of Health Research Career Development Award NS00848, and the Chicago Community Trust/Searle Scholars Program.

Correspondence should be addressed to Dr. Walters, Department of Physiology and Cell Biology, University of Texas Medical School at Houston, P.O. Box 20708 , Houston, TX 77225.

Copyright (C) 1988 Society for Neuroscience $0270-6474 / 88 / 083000-11 \$ 02.00 / 0$
}

been studied explicitly at the cellular level. Indeed, it has received very little attention at the behavioral level (Kimble, 1961). Although there has been some confusion in the use of the word "pseudoconditioning," this term can be precisely defined at the behavioral level so that it is clearly distinguished from sensitization and other classes of behavioral modification (see Discussion). Like sensitization, pseudoconditioning is produced nonassociatively by presentations of a strong, unconditioned stimulus (US). Whereas sensitization is defined as a quantitative enhancement of preexisting behavioral responses ("alpha responses") to a test stimulus, pseudoconditioning is defined as the appearance of novel (qualitatively different) test responses that resemble the US-evoked unconditioned responses (UR) produced during training.

Aplysia has proven very useful for the cellular analysis of both associative and nonassociative alterations in the magnitude of preexisting behavioral responses, but this relatively simple mollusk has not yet provided models for the analysis of learning involving the acquisition of qualitatively different responses to a test stimulus. Recently, we found that Aplysia has several distinct siphon responses that are used to direct defensive mantle secretions towards a site of threatening stimulation (Walters and Erickson, 1986). In this paper, we describe how a strong US applied to one part of the body can cause a qualitative transformation of the siphon response elicited by test stimulation of another part of the body, with the siphon response to the test stimulus changing to a response resembling that to the US. We classify this transformation as pseudoconditioning because the properties of the transformation in Aplysia closely resemble properties described in various examples of this relatively neglected class of behavioral modification. Moreover, we suggest that pseudoconditioning represents a fundamental and widespread form of response modifiability that may prove useful for analyzing neural mechanisms by which an organism's normal response selection rules are altered. Because the neural circuitry underlying siphon responses in Aplysia is relatively well known and accessible, specific hypotheses about mechanisms of pseudoconditioning in this system can be formulated and tested directly at the cellular level. Some of the results of this paper have been presented in abstract form (Erickson and Walters, 1986, 1987).

\section{Materials and Methods}

Aplysia californica (150-300 gm), supplied by Alacrity Marine Biological Services (Redondo Beach, CA) and Sea Life Supply (Sand City, CA), were kept in artificial seawater ("Instant Ocean") at $19^{\circ} \mathrm{C}$. Relatively constant body weight was maintained with meals of romaine lettuce twice a week. 
Unrestrained animals. The general training and testing procedures for the unrestrained animals have been described previously (Walters, 1987a). All test manipulations and scoring of siphon responses (Figs. 1, 2) were done "blind" by an observer who did not know the training history of the animal. The US used for training were $50-60 \mathrm{~mA}, 60 \mathrm{~Hz}, \mathrm{AC}$ shocks applied to the center of the anterior part of the head or to the center of the tail through hand-held dual-capillary electrodes pressed gently against the skin. Trains $(0.5 \mathrm{sec})$ were delivered at $5 \mathrm{sec}, 5 \mathrm{~min}$, or $10 \mathrm{~min}$ intervals. These stimuli did not leave perceptible lesions or cause contractions that persisted until the tests given $1 \mathrm{~d}$ after the US. Midbody test stimuli were von Frey hairs $(4.3 \mathrm{gm})$ or single $0.5 \mathrm{sec}$ trains of 10 $\mathrm{mA}, 60 \mathrm{~Hz}, \mathrm{AC}$ shock applied by light contact with a monopolar $\mathrm{Ag} /$ $\mathrm{AgCl}$ electrode. All test stimuli were delivered to a point marked with a knot of 00 suture thread near the midpoint of the base of one of the parapodia. Sutures were applied to each parapodium 3-5 d before testing, at the same time that the animal was parapodectomized to reveal the siphon (see Pinsker et al., 1973; Walters and Erickson, 1986).

Reduced preparation. The siphon, mantle, and gill, connected to the intact abdominal and head ganglia, were dissected out after anesthetizing the animal with isotonic $\mathrm{MgCl}_{2}$. The siphon was placed over a photocell, as shown in Figure 1. Restraining pins were only put into the anterior mantle region, permitting unrestricted siphon movement. The siphon was continuously perfused with filtered, aerated artificial seawater through a small cannula inserted into the musculature at its base, and stabilized with a small drop of "superglue" applied at the insertion into the skin. The chamber was perfused separately at a rapid rate and the perfusate sucked out through an outlet tube placed near the ink gland. The inlet and outlet tubes were positioned so that almost all the ink released by the US was drawn away from the siphon and photocell. Five to ten different nerves were drawn into suction electrodes and, after at least 1 $\mathrm{hr}$ perfusion, were tested for their threshold to elicit a siphon movement, using progressively greater DC currents in $100 \mathrm{msec}$ trains of $1 \mathrm{msec}$ pulses at $50 \mathrm{~Hz}$. Test stimuli were delivered to a nerve innervating the midbody region and the amplitude of the siphon response measured. The test nerve of choice was $p 8$ (see Kandel, 1979, for nomenclature), but if relatively stable, short-latency siphon responses could not be obtained from $\mathrm{p} 8$, other nerves innervating the midbody region were used (in order of priority, p8, p7, p6, and the anterior branch of $p 9$, which innervates the ipsilateral parapodium and not the tail). No obvious differences were observed among these nerves in their capacity to display pseudoconditioned or sensitized responses, and the different training groups included similar proportions of these test nerves. The formal test stimulus was then usually set at $105-125 \%$ of the threshold current. Animals that did not display 5 or more consecutive siphon responses in the 10-trial baseline block of tests were not included in the analysis. In 12 early experiments (evenly distributed among the different training groups), the test current intensity was set at $300 \%$ of threshold. The higher current, however, appeared to reduce the likelihood that test responses would be produced on at least 5 consecutive baseline trials, and so the remaining 42 experiments were conducted with the lower test currents. For all animals that were used-those that met the 5-trial criterion-there was no apparent difference between high- and lowcurrent levels in the response characteristics or in the alterations by pseudoconditioning or sensitization training. The US used in training was set at $200-300 \%$ of threshold and was applied to either a branch of $\mathrm{c} 2$ (which innervates the head) or to the posterior branch of $\mathrm{p} 9$ (which innervates the tail) in 3 trains $5 \mathrm{sec}$ apart. The US was applied midway between tests 10 and $11(30 \mathrm{sec}$ before test 11$)$. Three protocols were used: "habituation training," simple repetition of a test stimulus at 60 sec intervals; "sensitization training," application of a US that caused a UR of the same type as the alpha test response; and "pseudoconditioning training." application of a US that caused a UR opposite to that of the alpha test response. Statistical analysis of formal studies in the reduced preparation $(n=54$; Figs. 4,5$)$ involved 1 -tailed tests of explicit a priori hypotheses (see Results) based on pilot studies $(n=62)$.

\section{Results}

The siphon of Aplysia displays 3 qualitatively different responses to moderate or intense mechanical or electrical stimulation of different regions of the body (Walters and Erickson, 1986). Anterior stimulation causes the siphon to constrict completely and rotate forwards; posterior stimulation causes the siphon to flare open and rotate backwards; and midbody stimulation causes a longitudinal contraction, with partial constriction-the lumen of the siphon is narrowed, but not completely closed off (Figs. $1,2)$. The major question in these studies was whether a preexisting (alpha) siphon response to a midbody stimulus could be qualitatively transformed into a siphon response resembling either the response to anterior stimulation-a "head-type response," or the response to posterior stimulation-a "tail-type response" (Figs. 1, 2), by applying a US to the head or tail. In other words, would a strong US to the head or tail cause pseudoconditioning of siphon responses elicited by midbody stimulation?

\section{Long-term pseudoconditioning in the unrestrained animal}

Pseudoconditioning of siphon responses in the freely moving animal was examined in 3 studies, all of which involved application of strong shocks to the head or tail as the US and application of moderate-intensity midbody test stimuli $24 \mathrm{hr}$ later. The midbody test responses were scored in a blind procedure, using the 5-point scale illustrated in Figure 2 . In the first study $(n=8)$, the US was applied 10 times at 5 min intervals, using parameters similar to those used for classical conditioning of siphon responses (Carew et al., 1981, 1983). In the second study $(n=12)$, the US was applied 10 times at $5 \mathrm{sec}$ intervals, using parameters similar to those used to study long-term potentiation (Walters and Byrne, 1985) and site-specific sensitization (Walters, 1987a, b) in Aplysia. In the third study ( $n=$ 4), the latter procedure was repeated 3 times at 10 min intervals. These different training procedures were used to test whether protocols that are commonly used to produce long-term changes in Aplysia reflexes can also produce pseudoconditioning. The test stimulus to the midbody region in the first study was a von Frey hair, and in the next 2 studies it was weak electric shock. There were no differences in median siphon scores using thesc 2 types of test stimuli. In fact, the median scores and interquartile ranges for both the pretest and the $1 \mathrm{~d}$ test were exactly the same for the same groups in each of the 3 studies; thus, for brevity, data from each study are pooled for display and analysis (Fig. 2). Prior to training, all but 3 of the 24 animals displayed siphon responses that were neither head-like nor tail-like (siphon scores of 3). One day after training, the animals that had received the US to the head displayed siphon responses to the midbody test stimulus that were significantly more head-like than the test responses before training (Wilcoxon's signed-rank test: $n=12$, $t=7, p<0.02$ ), and animals that had received the US to the tail displayed responses that were significantly more tail-like ( $n$ $=12, t=0, p<0.005$ ). Not surprisingly, there were also significant differences on the $1 \mathrm{~d}$ tests between the animals that had received the head US and those receiving the tail US (MannWhitncy $U$ test; $U=9.5, p<0.001$ ). No signs of tissuc damage or tonic contraction were seen at the time of testing in these studies, suggesting that the blind was not compromised by cues from the US site.

\section{Pseudoconditioning and sensitization in the reduced preparation}

Although our investigations of the intact animal indicated that siphon responses can be pseudoconditioned, it is important for the analysis of underlying mechanisms to show that pseudoconditioning can be produced in a reduced preparation suitable for electrophysiological recording. The reduced preparation we have developed (Fig. 1) uses a photocell to monitor the amplitude and type of directional siphon responses, allowing quan- 


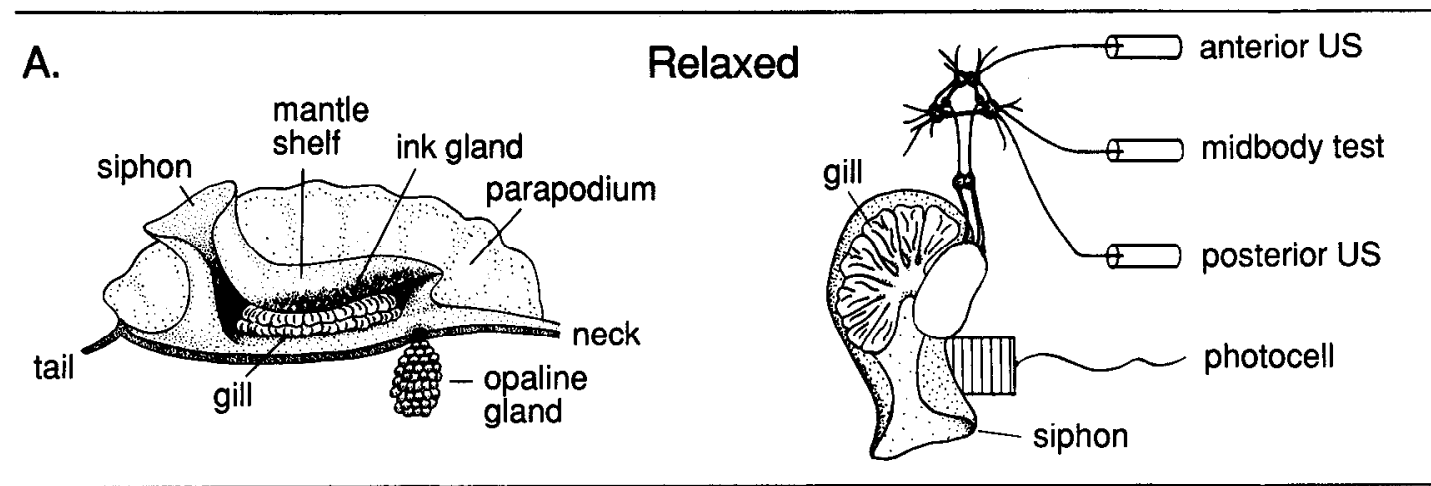

B. Flaring, Tail-type Response

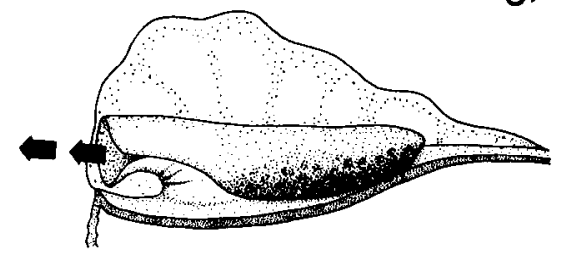

posterior stimulation
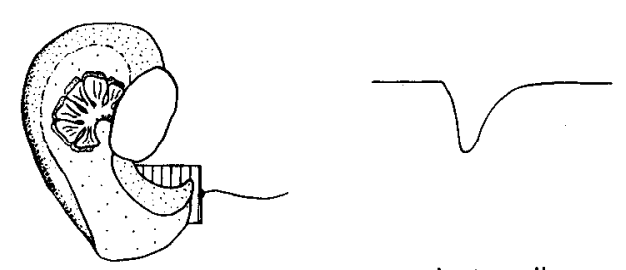

photocell

C. Constricting, Head-type Response
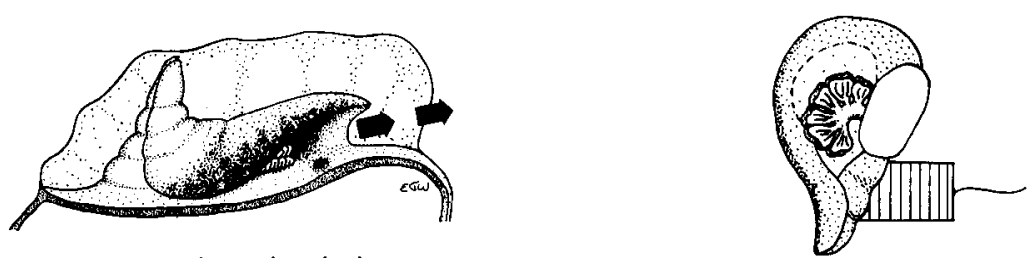

responses

anterior stimulation

Figure 1. Tail-type and head-type siphon reponses in the intact animal and reduced preparation. Left column, Views of the inside of the mantle cavity as it would appear in the intact animal if one could look through the right parapodium (not shown). The ink gland and opaline gland empty into the mantle cavity (the opaline gland is shown only in $A$; other internal organs are omitted). Right column, The mantle organs, ventral-side up, as arranged in the reduced preparation. The photocell is placed so that changes in breadth, but not in length, of the siphon are monitored. $A$, Relaxed position. The reduced preparation shows the suction electrodes used to deliver strong USs to anterior or posterior nerves and test stimuli to midbody nerves. $B$, Flaring, tail-type response to stimulation of posterior skin or nerve. The arrow indicates the caudally directed flow of seawater and defensive secretions from the mantle cavity. $C$, Constricting, head-type response to stimulation of anterior skin or nerve. In the intact animal this response directs the defensive secretions forwards (see Walters and Erickson, 1986).

titative measurement of pseudoconditioning and sensitization that is not subject to potential observer bias during data collection. Furthermore, the reduced preparation permits the use of nerve shocks as test and training stimuli, thus excluding the possibility that the pseudoconditioned responses merely reflect inadvertent, movement-induced reafferent stimulation from sites on the head or tail that might have been damaged or locally sensitized by the US (see Walters, 1987a, b).

We used different stimulus parameters in the reduced preparation than were used in the freely moving animal for 3 reasons. First, the effective lifetime of this reduced preparation was shorter than the $24 \mathrm{hr}$ retention interval used with the freely moving animals. Second, to monitor potential differences in the time course of sensitization and pseudoconditioning, we applied more frequent test stimuli (blocks of 10 tests at 1 min test intervals) to the reduced preparation. Third, in pilot studies we have noticed that very intense stimuli sometimes cause refiex suppression rather than sensitization or pseudoconditioning shortly after training (Krontiris-Litowitz et al., 1987; cf. Marcus et al., 1987). To minimize this short-term suppression, we reduced the effective intensity of the US by delivering only 3 US trains to the reduced preparation.

Figure 3 shows photocell recordings from animals given sensitization training and those given pseudoconditioning training. Because of the arrangement of the siphon on the photocell (Fig. 1), measurement of siphon movements is restricted to a single dimension, with constricting responses causing positive excursions, and flaring responses causing negative excursions on the chart record. Thus, head-type and tail-type siphon responses are distinguished by the sign of the photocell response. A limitation of this 1-dimensional monitoring arrangement is that characteristic midbody responses (which are primarily longitudinal contractions, producing movements orthogonal to the dimension monitored by the photocell) are not very distinctive: sometimes they are monophasic and weakly positive (a net constricting response) or weakly negative (a net flaring response), and sometimes they are multiphasic, with sequential responses of opposite types. To overcome this limitation, we examined only effects of training on short-latency alpha responses to midbody nerve stimulation that were reliably head-like or tail-like 
Flaring,
Tail-type
Response

Midbody
Response
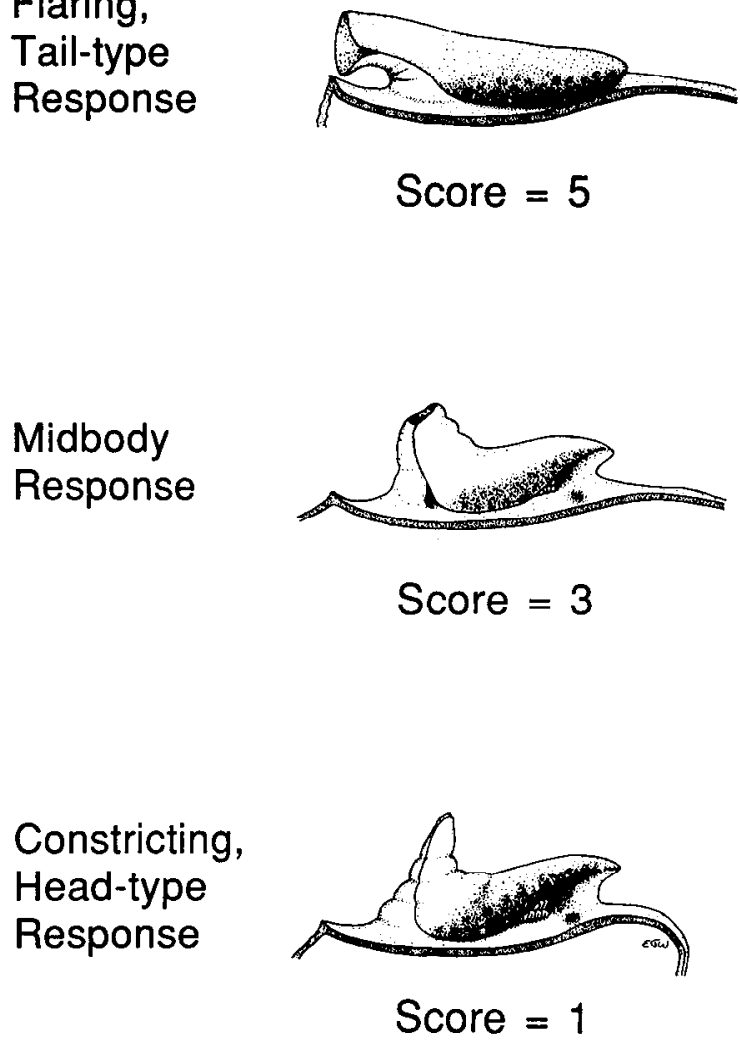

Score $=5$

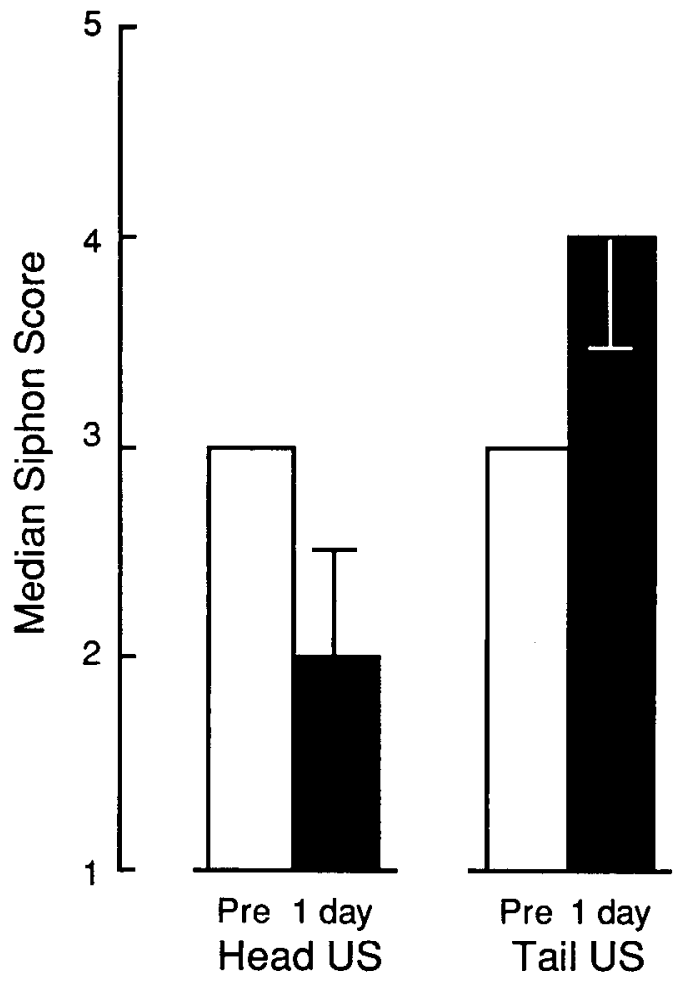

Figure 2. Pseudoconditioning of siphon responses in the intact animal. Left column, Responses corresponding to the extremes and to the midpoint of the siphon-scoring scale used by the "blind" observer. Scores of 4 and 2 were given to responses that were clearly tail-like or head-like, respectively, but were not as pronounced as those shown for scores of 5 and 1 . Right column, Median siphon scores ( \pm interquartile range) to midbody test stimulation before and $24 \mathrm{hr}$ after training with either a strong head US or a strong tail US.

and clearly larger than any secondary responses during the baseline trials. Whether the alpha response was head-like or tail-like was evaluated by the sign of the response on the chart record (see below), and confirmed by the subjective observations of the experimenter. These selection criteria provided a particularly demanding test for pseudoconditioning because pseudoconditioned responses could only be recognized in the photocell output if the alpha response actually reversed direction, i.e., converted from a head-like to a tail-like response or vice versa. Sensitization was then expressed as an increase in the amplitude of this alpha response (Fig. $3 A$ ), whereas pseudoconditioning was expressed as a reversal of the sign of the short-latency alpha response (Fig. 3B).

To compare pseudoconditioning and sensitization (from both anterior and posterior stimulation), we expressed the amplitude of the test responses as a percentage of the initial response amplitude (Fig. 4A). This normalization results in positive values for both constricting and flaring responses as long as the direction of the siphon response (i.e., the sign of the change from baseline measured by the photocell) is the same as the direction of the initial response. On the other hand, a change in direction of the response (from constricting to flaring or vice versa) yields a negative normalized value. The net result is that, regardless of the direction of the initial response, sensitization is expressed as a change in the positive direction and pscudoconditioning is expressed as negative normalized response values. It is important to note that, although this measuring system allows sensitization and pseudoconditioning to be expressed along a single continuum, the zero point represents a critical qualitative break in the nature of the behavioral response being measured. Positive responses (constriction) and negative responses (flaring) are distinct, mutually incompatible responses that presumably involve at least partially separate sets of muscles (as judged by differences in the sites of contraction; see Walters and Erickson, 1986). A control group receiving test stimuli alone-habituation training - was run to show that response reversal (pseudoconditioning) does not occur spontaneously or as a consequence of simply repeating the test stimuli.

On the basis of pilot studies in the reduced preparation, we tested 2 explicit hypotheses: (1) sensitization training (applying a US that produces a siphon UR of the same type as the alpha test response) would cause significantly larger test responses than would habituation training, and (2) pseudoconditioning training (applying a US that produces a siphon UR opposite to the alpha response) would, by reversing the direction of siphon movement, produce significantly smaller (negative) test responses than would habituation training. These hypotheses were evaluated by between-group comparisons of the average responses during trials 11-20 (immediately following the US) and 21-30 (after $60 \mathrm{~min}$ rest). To reduce the effects of variability among different animals, the average response during trials $11-20$ and $21-30$ in each animal was divided by the average response of that animal during baseline trials $1-10$, yielding a "test ratio" for that animal. Again, a negative value for the test ratio can only occur if, after the US, the test responses convert to the opposite response type-are pseudoconditioned. Figure $4 B$ shows the differences in mean test ratios among the 3 groups. During trials 11-20, there was significant sensitization and pseudoconditioning (sen- 


\section{A. Sensitization Training}

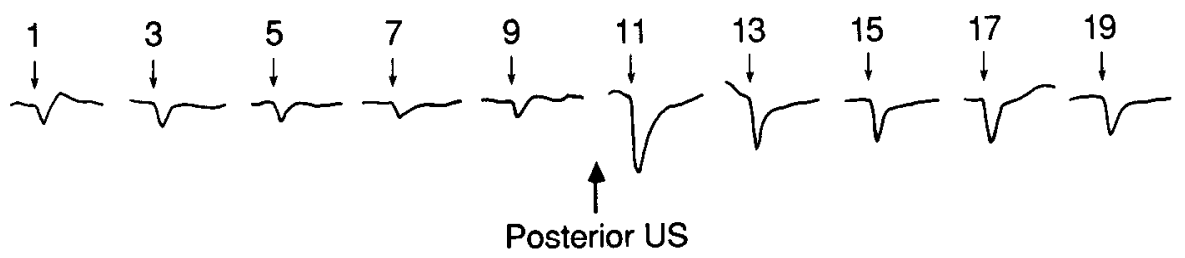

Figure 3. Sensitization and pscudoconditioning of midbody siphon responses monitored by photocell in the reduced preparation. $A$, Before and after sensitization training. The number of each test trial (only odd-numbered tests are shown) is displayed above the small arrow that indicates the midbody test stimulus (delivered to nerve p8). Top traces, The enhancement of alpha flaring responses by a posterior US (to nerve p9). Bottom traces, The enhancement of alpha constricting responses to the same midbody test stimulus by an anterior US (to nerve $c 2$ ). $B$, Before and after pseudoconditioning training. Top traces, The reversal of alpha flaring responses by an anterior US. Bottom traces, The reversal of alpha constricting responses by a posterior US. The test and training nerves were the same as in $A$.

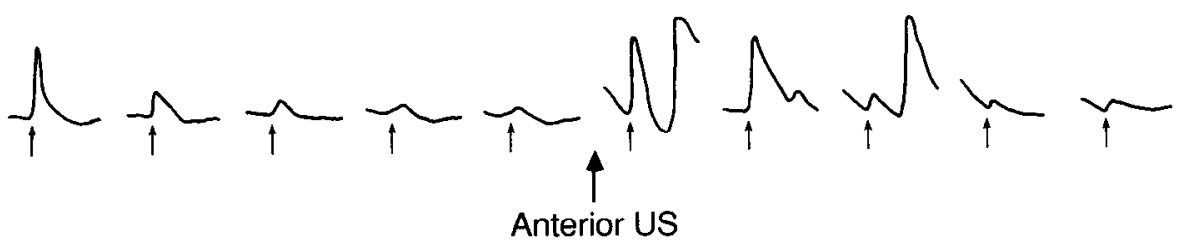

\section{B. Pseudoconditioning Training}
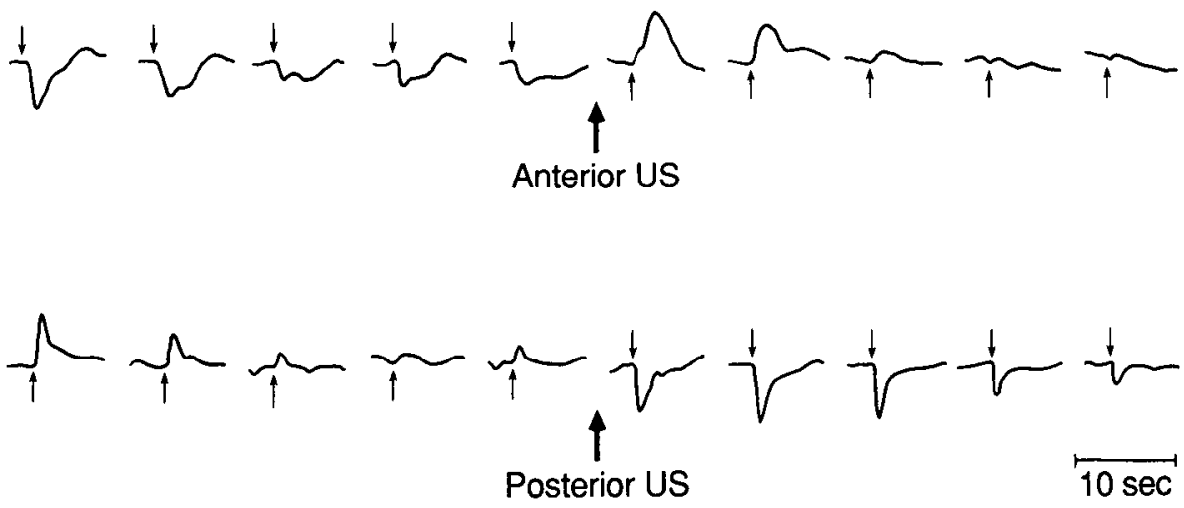

sitization vs habituation: $t_{19}=3.99, p<0.005$; pseudoconditioning vs habituation: $\left.t_{19}=2.62, p<0.01\right)$. Within-group comparisons yielded similar results. Comparison of the last baseline trial, 10 , to trial 11 showed that sensitization training caused significant enhancement of test responses $\left(t_{10}=5.72, p\right.$ $<0.005$ ), pseudoconditioning training caused a significant shift in the negative direction $\left(t_{10}=2.86, p<0.01\right.$, with 10 of 11 animals showing response conversions), and the habituation group was not significantly changed. Direct observation of the siphon responses indicated that the changes in response after pseudoconditioning were very similar to the qualitative changes seen in the intact animal.

There were no significant differences between the sensitization and pseudoconditioning groups and the habituation group after the $60 \mathrm{~min}$ rest. However, the fact that the siphon responses after sensitization training were greater than the responses after pseudoconditioning training following the $60 \mathrm{~min}$ rest $t_{19}=$ $2.10, p<0.05$ ) suggests that there may have been some weak residual effect of one or both forms of training.

To see if the type of midbody alpha response influenced the degree of pseudoconditioning or sensitization, we examined separately data from the animals displaying constricting alpha responses and from those displaying flaring alpha responses (Fig. 5). For each population, the US caused significant sensitization and pseudoconditioning of the midbody test ratios during trials 11-20 compared to habituation of the same response type (anterior US sensitization of constricting alpha responses: $t_{9}=2.68$, $p<0.025$; posterior US pseudoconditioning of constricting al- pha responses: $t_{9}=2.32, p<0.025$; posterior US sensitization of flaring alpha responses: $t_{8}=2.61, p<0.025$; anterior US pseudoconditioning of flaring alpha responses: $t_{9}=2.54, p<$ 0.025 ). After $60 \mathrm{~min}$ rest, one significant effect was observed, anterior pseudoconditioning of flaring alpha responses $\left(t_{8}=2.86\right.$, $p<0.025$ ). Although the constricting alpha responses (Fig. 5A) tended to display larger changes in the pseudoconditioned direction immediately after training than did the flaring alpha responses (Fig. 5B), and the flaring alpha responses (Fig. $5 B$ ) tended to display larger changes in the sensitized direction than did the constricting alpha responses (Fig. $5 A$ ), these apparent differences were not statistically significant in these relatively small groups. If real, these differences might reflect stronger effects of a US applied to the tail than to the head. Alternatively, the resting posture of the siphon (as measured by the photocell) may simply be closer to its maximally constricted posture than to its maximally flared posture, permitting greater changes in the flared than in the constricted direction.

Small, relatively long-lasting changes in the posture of the siphon were sometimes observed after US application in the reduced preparation. These were expressed as an incomplete return to baseline during trials 11-20. US-induced changes in siphon posture will be described elsewhere, because measurement of small changes in siphon position in the present experiments was confounded by interference from ink released by the US. Although most of the ink was rapidly washed out of the chamber, residual ink could shift the photocell trace slightly for several trials. This shift did not amount to more than $5-10 \%$ 


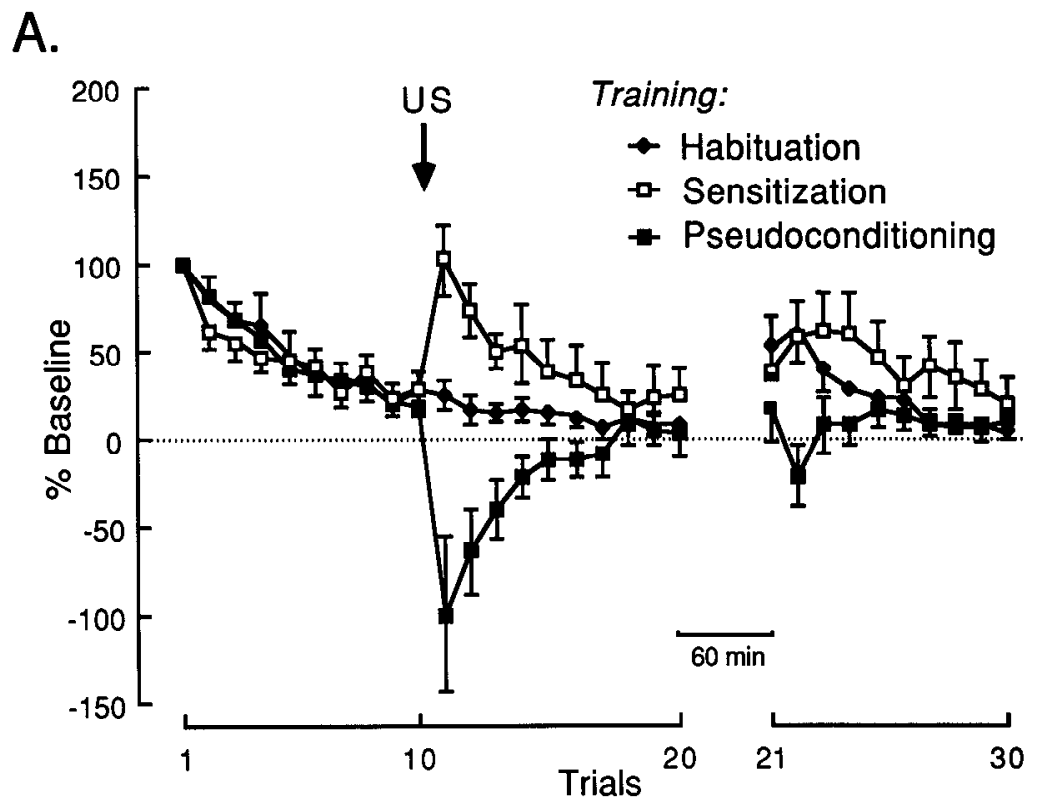

B.

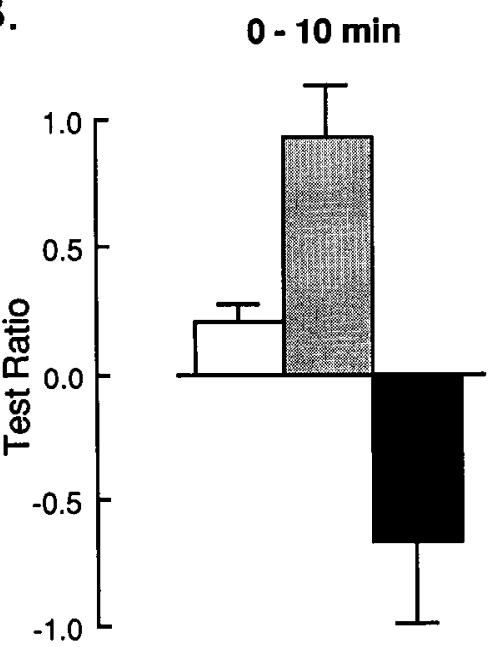

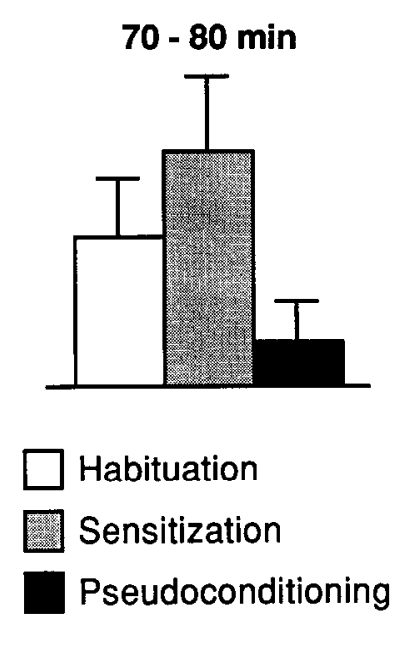

Figure 4. Effects of pseudoconditioning, sensitization, and habituation training in the reduced preparation. $A$, Mcan ( \pm SEM) response values on each trial. The initial baseline response, whether flaring or constricting, is plotted as $100 \%$. Because the arrangement of the siphon on the photocell restricts measurements to movements within a single dimension, negative values represent a conversion of the initial response type (from a flaring to a constricting response, or vice versa) and are directly correlated with the amplitude of the converted response. $B$, Mean ( \pm SEM) test ratios of each group for 10-trial blocks immediately after the US $(0-10 \mathrm{~min})$ and following a $60 \mathrm{~min}$ rest period $(70-80 \mathrm{~min})$. The test ratio for each animal is the mean response during the indicated 10-trial block divided by the mean response before the US (trials 1-10). of the maximal phasic response amplitude, and, when similar amounts of ink were artificially introduced into the chamber, caused little interference with the amplitude of the phasic responses. The incidence and apparent intensity of inking were the same in the sensitization and pseudoconditioning groups, so differential ink release cannot account for differences between these groups. It is very unlikely that changes in the resting posture of the siphon in the same direction as the UR would explain either pseudoconditioning of sensitization, since, by bringing the siphon closer to the response ceiling of the UR, such tonic contractions would reduce, rather than mimic, the phasic expression of pseudoconditioning and sensitization.

\section{Modification of anterior and posterior test responses}

The studies described above, and most previous studies of siphon response modifiability (e.g., Pinsker et al., 1973; Carew et al., 1981), used stimulation of the midbody region (which includes the siphon itself) as the test input. We wondered whether the observed modifications of midbody test responses represented a general pattern of defensive reflex plasticity in Aplysia or whether there were special properties of midbody stimuli that were not shared by stimuli applied to other sites on the body. To begin to answer this question, we applied the same weak test stimuli used previously for midbody stimulation either to a posterior pedal nerve (p9) or to one of the anterior cerebral nerves ( $\mathrm{c} 2$ ), and applied the strong US to the matching contralateral nerve. The results are shown in Figure 6. Siphon test responses to $\mathrm{c} 2$ stimulation were always constricting, and test responses to $\mathrm{p} 9$ stimulation were always flaring. The habituation curves for constricting and flaring responses elicited by anterior stimulation (Fig. 6A), posterior stimulation (Fig. $6 B$ ), and midbody stimulation (Fig. 5) were virtually indistinguishable during the first 20 stimuli. Although there appeared to be a tendency for the midbody test responses to display more dishabituation with rest than did anterior or posterior test responses, these differences were not statistically significant. Likewise, short-term sensitization was generally similar in these groups. Compared to animals receiving habituation training of the same type of siphon response, there was significant sensitization of $\mathrm{c} 2$-evoked siphon responses by a contralateral c2 US (Fig. $6 A ; t_{9}=2.21$, $p<0.05$ ) and of $\mathrm{p} 9$-evoked responses by a contralateral $\mathrm{p} 9$ US (Fig. $6 B ; t_{9}=2.41, p<0.025$ ). A lack of significant differences 

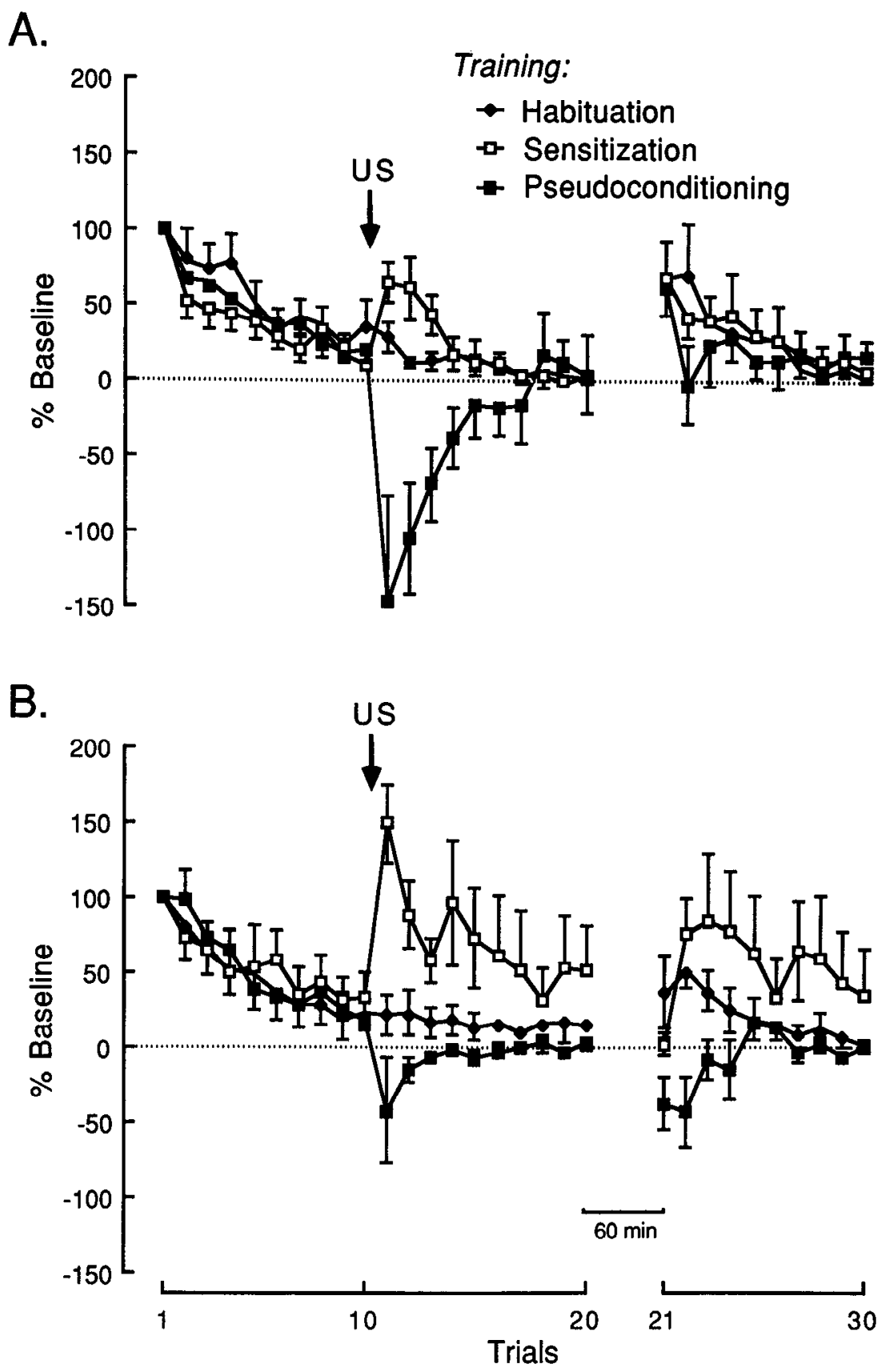

among the test ratios on trials $11-20$ of the groups given c2 test stimuli, $\mathrm{p} 9$ test stimuli, and midbody test stimuli indicated that short-term habituation and sensitization of siphon responses is similar whether the test responses are elicited by anterior, posterior, or midbody stimuli. However, in contrast to the results with midbody test stimuli, significant sensitization was still present after the $60 \mathrm{~min}$ rest in the groups given anterior or posterior test and training stimuli ( $\mathrm{c} 2$ responses: $t_{9}=1.91, p<0.05 ; \mathrm{p} 9$ responses: $t_{9}=2.18, p<0.05$ ).

\section{Pseudoconditioning can dominate sensitization}

In the present studies, "pseudoconditioning training" is defined as the application of a US that produces a UR opposite to the type of alpha response initially produced by the midbody test stimulus. This definition only specifies the training operations and not the outcome-whether or not pseudoconditioning of test responses actually occurs. Indeed, previous views of sensitization in Aplysia (e.g., Kandel and Schwartz, 1982) implied that a strong US applied to any point on the body should simply enhance (sensitize) the alpha siphon response, even when the UR and the initial alpha response are qualitatively different. Given the capacity of the siphon response system for sensitization, we were interested in assessing the relative strength of sensitization and pseudoconditioning under conditions in which pseudoconditioning can be expressed (conditions under which, by previous views, sensitization should have been expressed). This was done by comparing the incidences of pseudoconditioning and sensitization in experiments in which the midbody alpha response to the test stimulus was opposite to the UR measured on the photocell. We broadened the scope of this comparison as much as possible by including data from all the studies of pseudoconditioning undertaken in the reduced prep- 

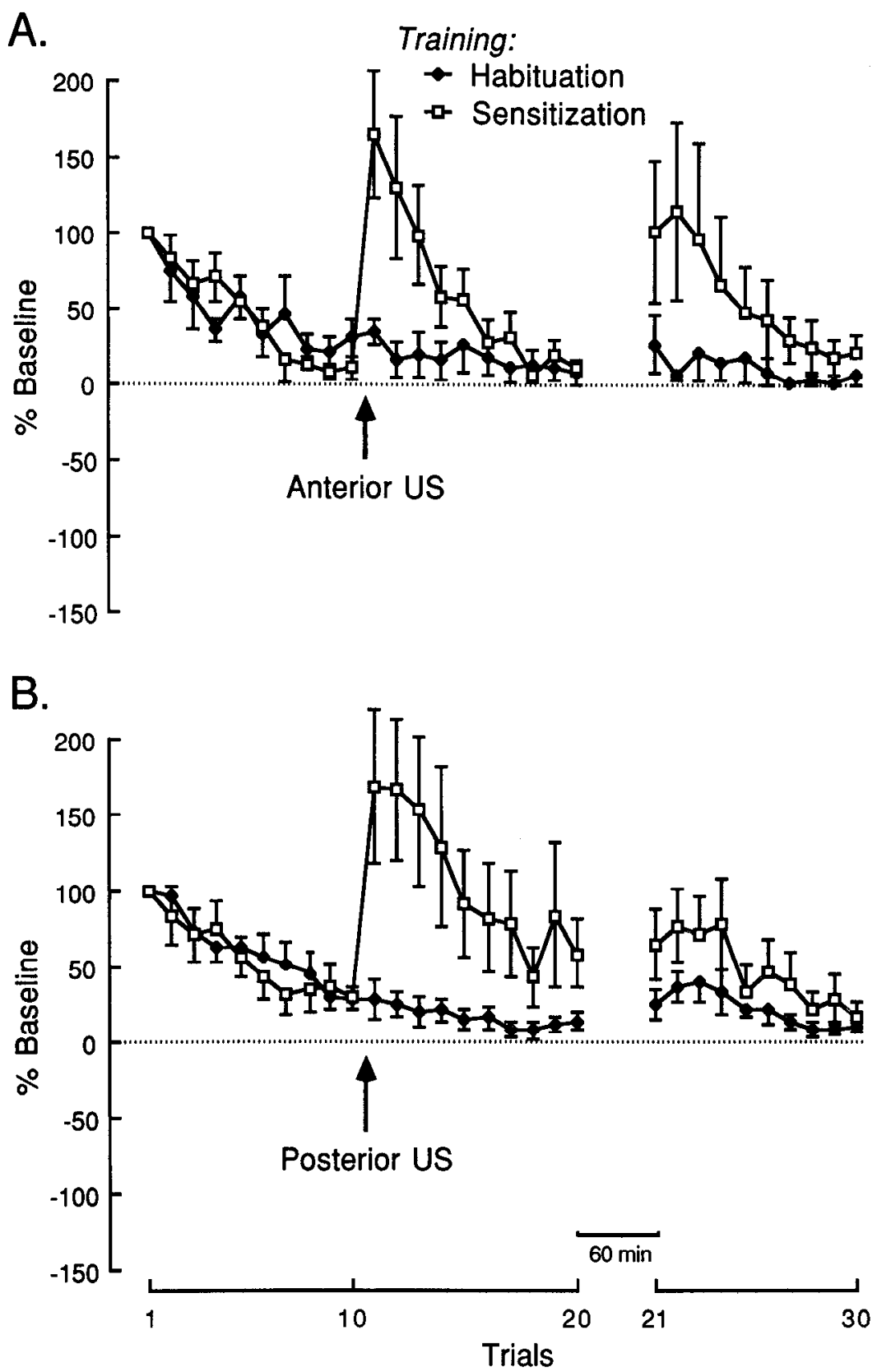

Figure 6. Sensitization and habituation of responses to anterior or posterior test stimuli. $A$, Effects on anterior (c2 stimulation) test responses of a US applied to the contralateral $\mathrm{c} 2$ nerve. $B$, Effects on posterior ( $\mathrm{p} 9$ stimulation) test responses of a US applied to the contralateral p9 nerve.

aration (Fig. 7). These included data illustrated in Figure 4 ( $n$ $=22$ ), data from various pilot experiments that used the same nerve stimuli but different test intervals and numbers of training trials $(n=35)$, and data from experiments similar to that of Figure 4, but with a mechanical US (strong tail-pinch or headpinch) and either a mechanical test stimulus (parapodial pinch; $n=13$ ) or an electrical test stimulus delivered to the skin (parapodial shock; $n=14$ ). Under each condition, a majority of the preparations showed some pseudoconditioning (clear reversal of the test response on at least one posttraining test), and 20 $40 \%$ of the preparations showed either no change or, more commonly, depression of the response without actual reversal. Given previous views of sensitization, it was particularly surprising to find only 1 of 84 preparations displaying any net sensitization of midbody alpha siphon responses (clear facilitation of the test response on at least one posttraining trial) by a US that caused the opposite siphon response. The nearly complete lack of net sensitization in these studies is striking since, as described above, these very siphon responses are readily sensitized by the same USs when the URs are of the same type as the alpha responses. Thus, when the alpha response and UR are of opposing types in this system, pseudoconditioning appears to dominate sensitization.

\section{Pseudoconditioning is not due to generalized sensory facilitation}

An important question is whether pseudoconditioned responses are determined by the nature of the US and UR, or instead reflect the unmasking of a latent, preexisting response tendency by mechanisms of general sensitization. In principle, pseudoconditioning might be caused by general facilitation of sensory pathways. By this argument, if a subpopulation of sensory neurons innervating the midbody test site, or with axons in the midbody test nerve, were frimarily connected to head-type or 


\section{A. Constricting Alpha Response, Posterior US}

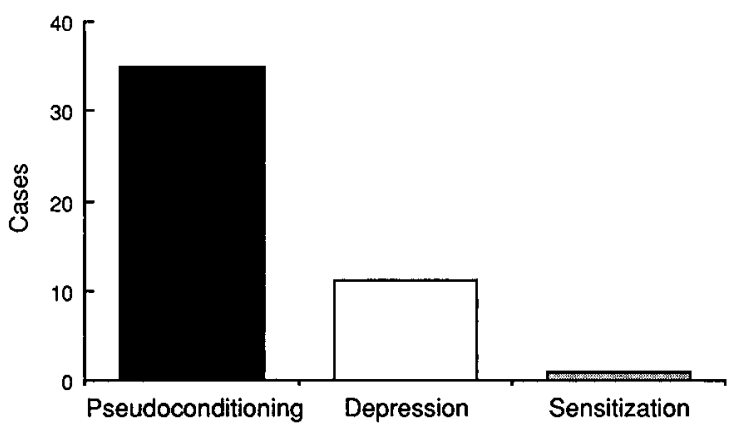

B. Flaring Alpha Response, Anterior US

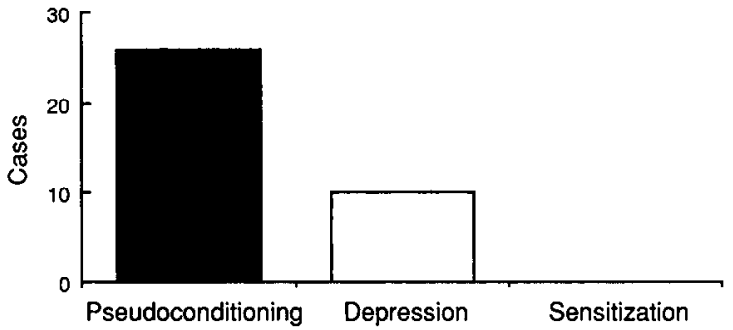

Figure 7. Prevalence of pseudoconditioning over sensitization in experiments in which the alpha midbody test response and the UR were opposite siphon response types. All cases indicated (each from a different animal) involved photocell monitoring of responses in reduced preparations. $A$, Effect of nerve $\mathrm{p} 9$ stimulation on initially constricting midbody responses. $B$, Effect of nerve $c 2$ stimulation on initially flaring midbody responses.

tail-type motor systems, and these connections were normally subthreshold, general facilitation of all sensory neurons by the US might unmask these latent connections, transforming a midbody-type siphon response to a head-type or tail-type response. This potential mechanism of response transformation cannot, however, explain the pseudoconditioning of both head-type and tail-type siphon responses. Random distribution between animals of the relative strength of latent connections to head-type or tail-type motor systems within the test pathway could explain pseudoconditioning in individual animals but not across a population of animals. An asymmetric distribution of latent connections favoring head-type or tail-type responses could explain pseudoconditioning of either head-type or tail-type responses, but not both.

To test this inference further, we asked whether siphon responses to midbody stimulation could be transformed to headtype or tail-type responses simpiy by increasing the intensity of the test stimulus (in effect, delivering a US to the test site). A very intense test stimulus should cause immediate increases in the effectiveness of activated sensory connections in the test pathway by means of temporal and spatial summation, and through persistent increases by both general and activity-dependent facilitation (Walters, 1987b). We examined 6 unrestrained animals for signs of head-like or tail-like responses to midbody stimulation over an exhaustive range of stimulus intensities (successive $0,4,8,40$, and $80 \mathrm{~mA}$ shocks delivered via a thin, insulated $\mathrm{Ag} / \mathrm{AgCl}$ electrode pressed against the middle of the base of the parapodium). The $80 \mathrm{~mA}$ shock caused clear tissue damage and copious inking. At the 3 lowest intensities, all animals displayed typical midbody responses, which received siphon scores of 3 (see Fig. 2). At the 2 highest intensities, all animals showed stronger longitudinal contraction than was seen at lower intensities, but none showed a head-type or tail-type siphon response and all, again, received siphon scores of 3 . When the series of shocks was repeated to the same midbody site $1 / 2 \mathrm{hr}$ later, there were, again, no clear head-type or tail-type responses to midbody stimulation in any of the animals, and all the siphon responses received scores of 3 . In summary, there were no significant differences in response type across a broad range of stimulus intensities in these 6 animals, suggesting the absence of latent head-type or tail-type response tendencies in these midbody pathways.

\section{Discussion}

The present studies provide the first demonstration of pseudoconditioning in a preparation suitable for investigation at the level of identified neurons. These studies also draw attention to a distinctive and widespread class of behavioral modifiability that has largely been overlooked by investigators of learning.

\section{Defining pseudoconditioning}

Since the coining of the term by Grether (1938), pseudoconditioning has generally been used as a label for qualitative changes in responses to test stimuli following unpaired exposure to a strong US. Most of the investigators who initially described pseudoconditioning (e.g., Harris, 1941; Grant, 1943a) considered it to be different from sensitization, but early definitions of pseudoconditioning as the nonassociative acquisition of a response to "a formerly inadequate stimulus" after US application (Grant and Dittmer, 1940; Harlow and Toltzien, 1940) did not clearly distinguish these 2 simple forms of learning. Some have added to this confusion by lumping all nonassociative influences of a US under the heading of either pseudoconditioning (e.g., Houston, 1986) or sensitization (e.g., Hintzman, 1978), and a few have suggested that pseudoconditioning can have associative components (e.g., Wickens and Wickens, 1942; Sheafor, 1975). The term "pseudoconditioning" will, however, only be useful to the extent that it refers to clearly defined, naturally occurring phenomena that are distinct from those defined by other categories of learning, such as sensitization and classical conditioning. We submit that the following definition, which is based on definitions used by Kandel and Spencer (1968) and Mackintosh (1974), satisfies these requirements and captures the views of the majority of investigators of pseudoconditioning. Pseudoconditioning is a nonassociative modification of behavior in which application of a US changes the quality of responses to stimuli other than the US, transforming these responses into ones resembling the UR or aspects of the UR. Sensitization, in contrast, is defined by most writers, including Kandel and Spencer (1968) and Mackintosh (1974), as a nonassociative enhancement of preexisting responses to a test stimulus following exposure to a US. Thus, sensitization refers to changes in response sensitivity and intensity, while pseudoconditioning refers to changes in response selection and topography.

\section{Properties of pseudoconditioning}

Our findings on the pseudoconditioning of midbody siphon responses in Aplysia, coupled with observations made in various other preparations, reveal properties implicit in the definition of pseudoconditioning, as well as additional properties that may apply to many examples of pseudoconditioning. These properties are as follows:

1. The pseudoconditioned response to a test stimulus is qual- 
itatively different from the alpha response to the same test stimulus.

2. The pseudoconditioned response is not produced by simply increasing the intensity of the test stimulus.

3. The pseudoconditioned response shows broad stimulus generalization.

4. The pseudoconditioned response does not require any interaction, temporally specific or otherwise, between the test stimuli and the US during acquisition.

These properties are exemplified in a classic study of pseudoconditioned escape responses in goldfish by Harlow (1939). They are displayed by pseudoconditioned responses to midbody stimulation in Aplysia as well, although the stimulus-generalization property will be described elsewhere (in brief, we have found that pseudoconditioned siphon responses are expressed to pressure, vibration, and photic stimuli). Most of these properties are apparent in phenomena observed in the frog (Franzisket, 1963), goldfish (Sears, 1934), cat (Harlow and Toltzien, 1940), and human (Grant and Meyer, 1941), and are similar to properties of "reflex dominance" described by Ukhtomsky and his followers (reviewed in Kandel and Spencer, 1968; Razran, 1971; Woody, 1982).

It is important to note that these behavioral properties can only be used to identify pseudoconditioning when the test stimuli selected produce overt alpha responses that are, initially, qualitatively different from the UR. Although this condition is often met in investigations of associative learning, qualitative response changes in control groups are rarely analyzed explicitly. The implicit occurrence of qualitative changes in control groups in associative studies of other gastropod mollusks, notably Pleurobranchaea (Mpitsos and Collins, 1975; Davis et al., 1980) and Hermissenda (Crow, 1983; see also Lederhendler et al., 1986), suggests that cellular mechanisms of pscudoconditioning may be available for comparative study in several neurophysiologically advantageous molluscan preparations.

\section{Mechanisms of pseudoconditioning}

Pseudoconditioning reflects a change in the normal response selection rules of the animal: after experiencing the US, it responds to diverse stimuli with UR-like responses, rather than with its normal alpha responses. One potential explanation of novel siphon response selection in Aplysia is that apparently "novel" responses are simply latent, preexisting responses to midbody stimuli that remain subthreshold until all sensory neurons, including the sensory neurons connecting to the head-type or tail-type siphon motor neurons, are facilitated during general sensitization. Although this mechanism might contribute to the appearance of either head-type or tail-type siphon responses after training, general facilitation of sensory ncurons (Carew et al., 1971) cannot explain the pseudoconditioning of both types of response. Moreover, in the freely moving animal, increasing the intensity of midbody test stimulation (which would tend to bring latent connections from that site above threshold) failed to change the quality of the alpha siphon responses.

Other potential mechanisms of pseudoconditioning cannot be critically evaluated using behavioral data alone, but can be framed in terms that will allow direct testing in the neural system controlling siphon responses. Two general classes of mechanism are of particular interest in light of current knowledge about Aplysia. First, pseudoconditioning might occur by selective facilitation of sensory neurons in the test pathway that synapse on URspecific motor elements. This could involve selective activation of head- or tail-specific facilitatory interneurons (see IIawkins et al., 1981) that only facilitate sensory terminals connecting to motor elements involved in head- or tail-type URs (cf. Hawkins et al., 1983; Clark and Kandel, 1984; Schwarz and Susswein, 1986). More elaborate versions of a mechanism for selective facilitation of UR-specific sensory inputs, involving special circuitry to link facilitator output to sensory terminals synapsing on motor elements underlying the UR (e.g., Krasne, 1984), are also possible.

The response specificity of pseudoconditioning can, perhaps, be more efficiently explained by a second type of mechanism that has been better established experimentally-selective facilitation of motor control circuitry specific to the UR, e.g., by an increase in the excitability of response-dedicated trigger neurons (Krasne and Cho Lee, 1988). Such changes could occur at various levels in the motor hierarchy, as long as the site of change is response-specific. For example, posterior nerve stimulation in Aplysia selectively increases the excitability of particular siphon motor neurons (Frost et al., 1985). Because these motor neurons are specifically involved in tail-type flaring responses (E. T. Walters and M. T. Erickson, unpublished observations), an increase in their excitability would be expected to contribute to the appearance of pseudoconditioned siphon responses to diverse test stimuli after strong tail stimulation. US-evoked enhancement of excitability of response-specific motor elements also occurs in the ink motor neurons of Aplysia after a noxious US (Carew and Kandel, 1977), and may be reflected in other preparations as well, e.g., in neurons within motor areas of the cat brain (Brons and Woody, 1980; Matsumura and Woody, 1982); in a number of less direct studies of motor area plasticity that have been used to support Ukhtomsky's hypothesis of reflex dominance (reviewed in Razran, 1971; Woody, 1982); and in studics indicating sites of hyperexcitability downstream from afferent neurons that contribute to facilitated spinal reflexes after noxious stimulation (e.g., Woolf and McMahon, 1985).

These and various other potential mechanisms of pseudoconditioning are not mutually exclusive, and might normally operate in parallel and/or in series with the mechanisms of general sensitization already described in Aplysia sensory neurons. Indeed, it seems likely that a US applied alone will trigger several parallel mechanisms (cf. Groves and Thompson, 1970) that can be expressed behaviorally as (1) general sensitization, (2) site-specific sensitization, or (3) pseudoconditioning, depending on the behavioral test selected. If the neural mechanisms underlying pseudoconditioning and sensitization are at least partially separate, an important question concerns how these processes interact. At the behavioral level, our results show an apparent dominance of sensitization by pseudoconditioning in the midbody siphon response system when the initial alpha response is opposite to the UR (Fig. 7). This apparent dominance might be explained by US suppression of mechanisms of sensitization in those midbody sensory neurons or their terminals connecting to motor pathways that can oppose the UR. Alternatively, the suppression may not be of the processes of sensitization themselves but of the performance of incompatible motor responses, perhaps by reciprocal inhibition among the involved motor subsystems.

Theoretical alternatives such as these can be clearly defined and directly tested in identified cells within the siphon response system. This system will also allow examination of interactions between the processes underlying pseudoconditioning and associative plasticity. Since siphon responses display both asso- 
ciative alpha conditioning (Carew et al., 1981, 1983) and nonassociative pseudoconditioning, this system should allow a test of the hypothesis that classical conditioning of novel responses (stimulus-response learning) can be achieved by combining pseudoconditioning mechanisms with alpha conditioning mechanisms (Erickson and Walters, 1986).

\section{References}

Brons, J. F., and C. D. Woody (1980) Long-term changes in excitability of cortical neurons after Pavlovian conditioning and extinction. $J$. Neurophysiol. 44: 605-615.

Carew, T. J., and E. R. Kandel (1977) Inking in Aplysia californica. III. Two different synaptic conductance mechanisms for triggering central program for inking. J. Neurophysiol. 40:721-734.

Carew, T. J., V. F. Castellucci, and E. R. Kandel (1971) An analysis of dishabituation and sensitization of the gill-withdrawal reflex in Aplysia. Int. J. Neurosci. 2: 79-98.

Carew, T. J., E. T. Walters, and E. R. Kandel (1981) Classical conditioning in a simple withdrawal reflex in Aplysia californica. J. Ncurosci. I: $1426-1437$.

Carew, T. J., R. D. Hawkins, and E. R. Kandel (1983) Differential classical conditioning of a defensive withdrawal reflex in Aplysia californica. Science 219: 397-400.

Clark, G. A., and E. R. Kandel (1984) Branch-specific heterosynaptic facilitation in Aplysia siphon sensory cells. Proc. Natl. Acad. Sci. USA 81: 2577-2581.

Crow, T. (1983) Conditioned modification of locomotion in Hermissenda crassicornis: Analysis of time-dependent associative and nonassociative components. J. Neurosci. 3: 2621-2628.

Davis, W. J., J. Villet, D. Lee, M. Rigler, R. Gillette, and E. Prince (1980) Selective and differential avoidance learning in the feeding and withdrawal behavior of Pleurobranchaea californica. J. Comp. Physiol. 138: 157-165.

Erickson, M. T., and E. T. Walters. (1986) Pseudoconditioning, alpha conditioning, and stimulus-response learning in Aplysia. Soc. Neurosci. Abstr, 12: 398.

Erickson, M. T., and E. T. Walters (1987) Pseudoconditioning, sensitization, and habituation of directional siphon responses in Aplysia. Soc. Neurosci. Abstr. 13: 815.

Franzisket, L. (1963) Characteristics of instinctive behaviour and learning in reflex activity of the frog. Anim. Behav. 11: 318-324.

Frost, W. N., G. A. Clark, and E. R. Kandel (1985) Changes in cellular excitability in a new class of siphon motor neurons during sensitization in Aplysia. Soc. Neurosci. Abstr. 11: 643.

Grant, D. A. (1943a) The pseudo-conditioned eyelid response. J. Exp. Psychol. 32: 139-149.

Grant, D. A. (1943b) Sensitization and association in eyelid conditioning. J. Exp. Psychol. 32: 201-212.

Grant, D. A., and D. G. Dittmer (1940) A tactile generalization gradient for a pseudo-conditioned response. J. Exp. Psychol. 26: 404-412.

Grant, D. A., and H. I. Meyer (1941) The formation of generalized response sets during repeated electric shock stimulation. J. Gen. Psychol. 24: 21-38.

Grether, W. F. (1938) Pseudo-conditioning without paired stimulation encountered in attempted backward conditioning. J. Comp. Psychol 25: 91-96.

Groves, P. M., and R. F. Thompson (1970) Habituation: A dualprocess theory. Psychol. Rev. 77: 419-450.

Harlow, H. F. (1939) Forward conditioning, backward conditioning, and pseudo-conditioning in the goldfish. J. Genet. Psychol. 55: 4958 .

Harlow, H. F., and F. Toltzien (1940) Formation of pseudo-conditioned responses in the cat. J. Gen. Psychol. 23: 367-375.

Harris, J. D. (1941) Forward conditioning, backward conditioning, and pseudo-conditioning, and adaptation to the conditioned stimulus. J. Exp. Psychol. 28: 491-502.

Hawkins, R. D., and E. R. Kandel (1984) Is there a cell-biological alphabet for simple forms of learning? Psychol. Rev. 91: 375-391.

Hawkins, R. D., V. F. Castellucci, and E. R. Kandel (1981) Interneurons involved in mediation and modulation of gill-withdrawal reflex in Aplysia. II. Identified neurons produce heterosynaptic facilitation contributing to behavioral sensitization. J. Neurophysiol. 45: 315326.

Hawkins, R. D., T. W. Abrams, T. J. Carew, and E. R. Kandel (1983)
A cellular mechanism of classical conditioning in Aplysia: Activitydependent amplification of presynaptic facilitation. Science 219:400 404.

Hintzman, D. L. (1978) The Psychology of Learning and Memory, Freeman, San Francisco.

Houston, J. P. (1986) Fundamentals of Learning and Memory, Harcourt, Bracc, Jovanovich, New York.

Kandel, E. R. (1976) Cellular Basis of Behavior: An Introduction to Behavioral Neurobiology, Freeman, San Francisco.

Kandel, E. R. (1979) Behavioral Biology of Aplysia, Freeman, San Francisco.

Kandel, E. R., and J. H. Schwartz (1982) Molecular biology of learning: Modulation of transmitter release. Science 218: 433-444.

Kandel, E. R., W. A. Spencer (1968) Cellular neurophysiological approaches in the study of learning. Physiol. Rev. 48: 65-134.

Kimble, G. A. (1961) Hilgard and Marquis' Conditioning and Learning, Appleton-Century-Crofts, New York.

Krasne, F. B. (1984) Physiological analysis of learning in invertebrates. In Cortical Integration: Basic, Archicortical, and Cortical Association levels of Neural Integration, F. Reinoso-Suarez and C. Ajmone-Marsan, cds., pp. 53-76, Raven, New York.

Krasne, F. B., and S. Cho Lee (1988) Response-dedicated trigger neurons as control points for behavioral actions: Selective inhibition of lateral giant command neurons during feeding in crayfish. J. Neurosci. (in press).

Krontiris-Litowitz, J. K., M. T. Erickson, and E. T. Walters (1987) Central suppression of defensive reflexes in Aplysia by noxious stimulation and by factors released from body wall. Soc. Neurosci. Abstr. 13:815.

Lederhendler, I. I., S. Gart, and D. L. Alkon (1986) Classical conditioning of Hermissenda: Origin of a new response. J. Neurosci. 6 : 1325-1331.

Mackintosh, N. J. (1974) The Psychology of Animal Learning, Academic, London.

Marcus, E. A., T. G. Nolen, C. H. Rankin, and T. J. Carew (1987) Behavioral dissociation of dishabituation, sensitization, and inhibition in the siphon withdrawal reflex of adult Aplysia. Soc. Neurosci. Abstr. 13: 816.

Matsumura, M., and C. D. Woody (1982) Excitability changes of facial motoneurons of cats related to conditioned and unconditioned facial motor responses. In Conditioning: Representation of Involved Neural Functions, C. D. Woody, ed., pp. 451-457, Plenum, New York.

Mpitsos, G. J., and S. D. Collins (1975) Learning: Rapid aversive conditioning in the gastropod mollusk Pleurobranchaea. Science 188 : 954-957.

Pinsker, H. M., W. A. Hening, T. J. Carew, and E. R. Kandel (1973) Long-term sensitization of a defensive withdrawal reflex in Aplysia. Science 182: 1039-1042.

Razran, G. (1971) An East-West Synthesis of Learned Behavior and Cognition, Houghton Mifflin, Boston.

Schwarz, M., and A. J. Susswein (1986) Identification of the neural pathway for reinforcement of feeding when Aplysia learn that food is inedible. J. Neurosci. 6: 1528-1536.

Sears, R. S. (1934) Effect of optic lobe ablation on the visuomotor behavior of goldfish. J. Comp. Psychol. 17: 233-265.

Sheafor, P. (1975) "Pseudoconditioned" jaw movements of the rabbit reflect associations conditioned to contextual background cues. J. Exp. Psychol. [Anim. Behav.] 104: 245-260.

Walters, E. T. (1987a) Site-specific sensitization of defensive reflexes in Aplysia: A simple model of long-term hyperalgesia. J. Neurosci. 7 : $400-407$.

Walters, E. T. (1987b) Multiple sensory neuronal correlates of sitespecific sensitization in Aplysia. J. Neurosci. 7: 408-417.

Walters, E. T., and J. H. Byrne (1985) Long-term enhancement produced by activity-dependent modulation of Aplysia sensory neurons. J. Neurosci. 5: 662-672.

Walters, E. T., and M. T. Erickson (1986) Directional control and the functional organization of defensive responses in Aplysia. J. Comp. Physiol. A 159: 339-351.

Wickens, D. D., and C. D. Wickens (1942) Some factors related to pseudo-conditioning. I. Exp. Psychol. 31: 518-526.

Woody, C. D. (1982) Learning, Memory, and Higher Function: A Cellular View, Springer-Verlag, New York, Heidelberg.

Woolf, C. J., and S. B. McMahon (1985) Injury-induced plasticity of the flexor reflex in chronic decerebrate rats. Neuroscience 16:395404. 